\title{
Effect of grain shape on the dynamics of granular materials in 2D rotating drum
}

\author{
Nicolas Preud'homme ${ }^{1, *}$, Eric Opsomer ${ }^{1}$, Nicolas Vandewalle ${ }^{1}$, and Geoffroy Lumay ${ }^{1}$ \\ ${ }^{1}$ GRASP, Department of Physics, ULiège, B-4000 Liège, Belgium.
}

\begin{abstract}
We experimentally investigate the effect of the grain shape on the flow of granular material. The grain shape is modified to highlight the effect of grain circularity on granular flow in a 2D rotating drum. Using a laser cutter, we create particles with decreasing circularity. We observe that the effect of grain shape depends on the rotation speed of the drum. For high rotation speed, granular flow is influenced by the packing's dilatancy whereas, at low rotation speed, packing fraction seems to influence flowing dynamics. We link these two measurements to grain shape in order to explain its effect on granular flow.
\end{abstract}

\section{Introduction}

The rotating drum is often chosen in order to study the dynamics of granular materials. One example is the work performed by Espiritu et al. [1] who used this tool in a very practical way to characterize the flow of powders used in Laser Powder Bed Fusion (LPBF) processes. Compared to other techniques widely used to reproduce granular flow, the advantages of the rotating drum are that it is a closed system and that the flow rate can be finely adjusted with the rotation speed of the drum. It has been shown that depending on the rotation speed, different flow regimes appear in the rotating drum [2-4]. At low rotation speed, the flow is in a slipping regime. At intermediate rotation speed, the granular material is effectively flowing in a regime called cascading regime and finally, at high rotating speed, all the grains are ejected against the drum which is known as centrifuging regime. In the cascading regime, it has been shown that certain parameters strongly affect the flow of granular materials. Among all these parameters, cohesion seems to play an important role on granular flow $[5,6]$. It has also been shown that granular shape can influence granular flow. Experimental studies in rotating drums indicate significant differences between the flow of perfectly smooth spheres and angular grains such as cuboids [7]. Furthermore, granular shape seems also to influence the discharge in hoppers where spheres appear to flow more freely whereas irregular grains block at the hopper outlet [8]. Even in numerical DEM simulations of non spherical grain flows, similar results are obtained (see [9] for an in-depth description of DEM non-spherical grains implementation and [10] for a complete review).

In another experimental study using the rotating drum, Olson et al.[11] showed that changing the shape of the grains leads to counter-intuitive result as the irregular grains arrange in a more dilute packing but still form a very stable granular pile. However, the shape of the grains is not quantified and these results are therefore hardly comparable to other studies. In general, the effect of grain shape on granular flow is still not quantified.

The aim of this experimental work is to introduce a parameter quantifying the irregularity of the grains and study its effect on the granular dynamics.

\section{Experimental setup}

All parts of the rotating drum as well as the grains it contains have been cut out from $3 \mathrm{~mm}$ thick Plexiglas sheets using a laser cutter (Trotec Speedy 100). With the parameters required to cut into $3 \mathrm{~mm}$ thick Plexiglas, the cut thickness has been measured at approximately $0.5 \mathrm{~mm}$. The drum is composed of a $20 \mathrm{~cm}$ inner diameter annular ring fixed to a black circular plate. The grains are inserted in the annular ring and maintained by a transparent Plexiglas front plate in order to take images of the granular dynamics. In order to avoid grain blockage between the back and the front plate, a thin gap size has been fixed to $0.5 \mathrm{~mm}$ to let the grains move as freely as possible. Finally, the back plate is bolted to a stepper motor for which the rotation speed is set with a control unit (see Figure 1).

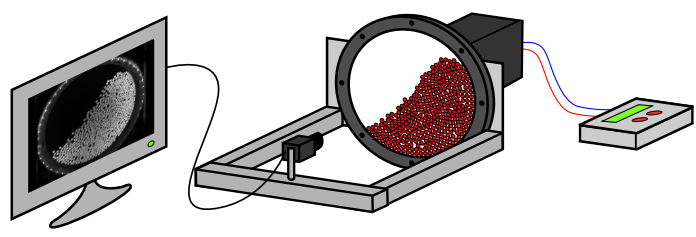

Figure 1. Schematic representation of the experimental setup consisting of a drum and grains in Plexiglas cut with a laser cutter. The drum is rotated by a stepper motor and rotation speed is set with a control unit. Images are taken with a monochromatic Thorlabs camera.

*e-mail: n.preudhomme@uliege.be

A video is available at https://doi.org/10.48448/md56-jy83 


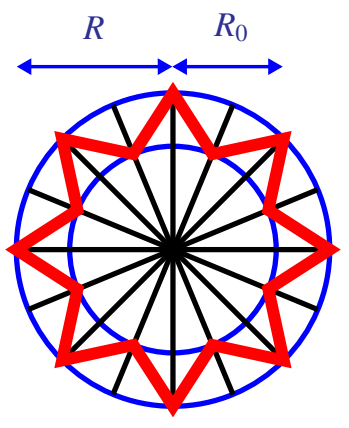

$\frac{R}{R_{0}}=\frac{3}{2}$

Figure 2. Design of the grains (in red) cut in Plexiglas. Two concentric circles are drawn, the outer one with a radius $R$ and the inner one with a radius $R_{0}$. The grains are then octagonal stars inscribed between these two circles. The higher the radius ratio, the higher the irregularity of the grains. The surface of the grain is kept constant for each shape.

Table 1. Parameters of the 2D grains employed for the study of the shape effect on the granular dynamics and their parameters. $R$ being the outer radius of the shape, $R_{0}$ the inner radius and $C$ its circularity.

\begin{tabular}{llll}
\hline & $2 R(\mathrm{~mm})$ & $\frac{R}{R_{0}}$ & $C$ \\
\hline 5.3 & 1 & 1.00 \\
& 6 & $6 / 5$ & 0.89 \\
& 6.65 & $3 / 2$ & 0.68 \\
\hline
\end{tabular}

The grains were cut in bright colors in order to increase contrast with the back. The shape of the grains has been designed as shown on Figure 2. In order to emphasize the effect of grain shape, different grain shapes were studied with growing irregularity. The irregular grains are eight pointed stars whose dimensions are defined by two concentric circles with radii $R_{0}$ and $R$ respectively for inner and outer circles and $R_{0}<R$ as shown on Figure 2. By varying the radius of the inner circle, one can create grains with different circularity $C$. The circularity is defined as the ratio between the perimeter of a circle with an equivalent surface and the real perimeter of the grain. As the surface has been kept constant for every different shape, the circularity $C$ is simply the ratio between the perimeter of the circular shape and the perimeter of the irregular shape. The circular shape has also been added for a complete comparison. The different parameters of the grains used for this study are summarized in Table 1. These grains are then inserted between the two Plexiglas plates. For all the experiments, the degree of filling of the drum has been set by adjusting the height of the granular packing at rest in the drum to the drum radius. This way, an apparent filling fraction of $50 \%$ is insured for every experiment. The rota-

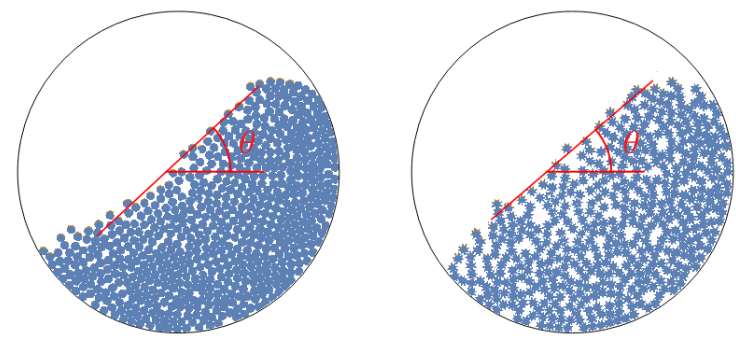

Figure 3. Measurement of the dynamic angle of repose $\theta$ for the disks (left) and the most irregular grains (right) on one binarized frame captured. A linear fit of the highest pixels detected on the surface (in orange) is calculated and the regression parameters directly give the desired angle. This method is repeated on each frame of the footage. The results are then averaged to obtain $\theta$.

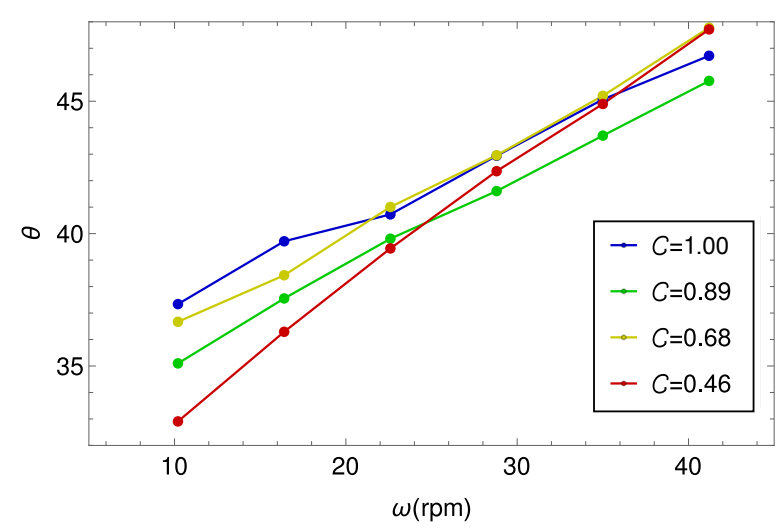

Figure 4. Dynamic angle of repose of the granular flow surface versus the rotation speed of the drum for different grain shapes described in Table 1.

tion speed is varied between $10 \mathrm{rpm}$ and $60 \mathrm{rpm}$ which lie within the limits of the cascading regime for our setup.

The Thorlabs monochromatic camera finally captures the motion of the grain inside the $2 \mathrm{D}$ cell at 20 frames per second for $40 \mathrm{~s}$.

\section{Results and discussion}

Once acquired, the images are binarized using a pixel value threshold. The dynamic angle of repose is then determined as shown on Figure 3 via a linear regression of the surface points near the central region of the drum, for each frame captured. By only considering the central part of the surface, we can avoid its non-linear parts which could appear when it becomes S-shaped. With the regression parameters, one obtains the dynamic angle of repose at the current frame. This operation is repeated for the whole 40-second video and the mean dynamic angle of repose $\theta$ is then calculated. The error of that measurement is estimated with the standard deviation and is typically of the order of a few degrees.

Figure 4 shows the measured $\theta$ values for different rotating speeds and grain shapes. At low rotation speed, the dynamic angle of repose is larger for circular particles than for highly irregular particles. Once the rotation speed is in- 


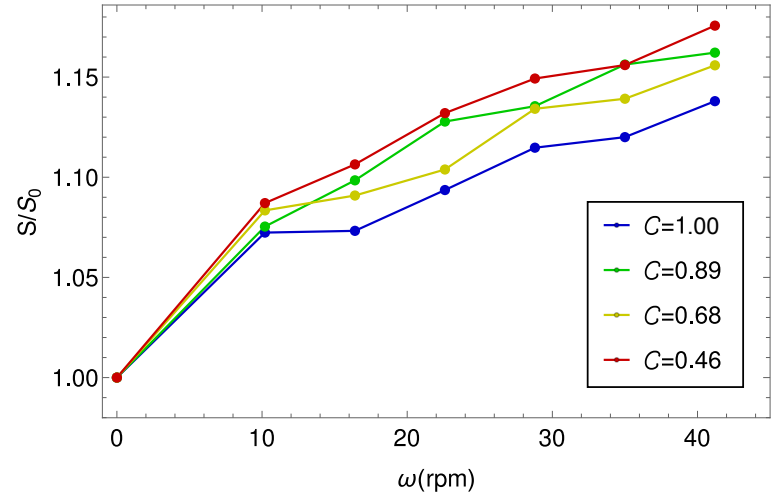

Figure 5. Dilatancy $S / S_{0}$ of the granular bed once rotated at a rotation speed $\omega$, for the different grain shapes.

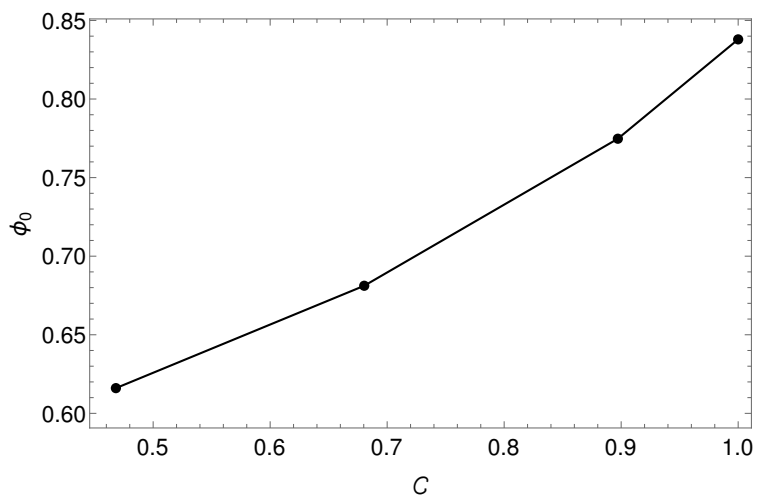

Figure 6. Packing fraction at rest $\phi_{0}$ of the different shapes defined by their circularity $C$.

creased, that tendency seems to invert: the highest angle is measured for the irregular grains. That second observation is consistent with the results obtained in experimental and numerical studies investigating the effect of grain shape $[8,10]$. However, this transition is quite surprising as we expected the granular pile of irregular grains to display a higher angle at low rotation speed. Indeed, if particles are only weakly agitated, they may form a strong granular packing as they interlock. On the other hand, if $\omega$ is large, one would expect the granular packing to be more brittle as those strong bonds begin to break and one would thus observe a lower angle for irregular grains. The opposite is observed and might be interpreted with the measurement of the dilatancy of the granular packing.

The dilatancy is defined as the ratio between the surface of the granular packing in motion $S$ divided by its surface at rest $S_{0}$. Figure 5 shows the dilatancy measured for the different grains shapes as a function of the rotation speed. We first observe that dilatancy increases for every shape. It is a well-known fact that sheared granular media composed of spherical grains tend to expand in order to flow and theory has been developed quite a long time ago [12]. The same phenomenon has been observed with irregular grains [13]. However, we observe that the rate at which the dilatancy increases with $\omega$ depends on the shape of the grains: when the rotation speed is increased, the granular packing composed of the most irregular particles seems to expand faster than those composed of nearly circular grains.

To summarize, irregular grains display, at high rotation speed, a larger dilatancy and a higher dynamic angle of repose. This result might be interpreted by the fact that dilatancy acts as a momentum damper [11]. As the granular packing expands, the grains in the flowing layer are more easily trapped in the upward stream of grains constituting the solid phase of the granular packing in the drum. On the contrary, taking the case of circular grains, the smaller dilatancy implies that the grains in the flowing layer easily flow on the solid phase underneath them. This finally results in a smaller dynamic angle of repose.

This effect of the dilatancy only becomes significant at high rotation speed. It means that another effect is behind the high values of $\theta$ at low rotation speed and low dilatancy. This effect might be given by the measurement of the packing fraction at rest $\phi_{0}$ of the different shapes shown on Figure 6. We measured the packing fraction as the ratio between the total surface of all the grains at rest divided by $S_{0}$. We observe that the packing fraction of the circular grains is much larger than the one for irregular grains. This implies that, at low rotation speed where the system has the time to relax and come to a rest, the most circular grains reach the highest angle of repose due to their higher packing fraction. This result is in agreement with experimental results from Olson et al. [11].

\section{Conclusion}

In this study, we show how the grain shape affects the granular dynamics. To put this effect in evidence, we measure the dynamic angle of repose as a function of the rotation speed of the drum. When varying the shape of the grains, we observe that $\theta$ does not increase with growing interlocking but that the latter favors shear driven expansion of the bulk. Analyzing both $\theta$ and the dilatancy brings us to the conclusion that two flowing regimes exist when varying the shape of the grains. At high rotation speed, the high angle measured for the most irregular grains comes from the expansion of the granular packing measured by the dilatancy. The empty spaces created by rotation in the granular material act as momentum dampers as irregular grains are trapped in those holes and immediately brought back to the top of the flowing layer. On the other side, at low rotation speed, the dynamic angle of repose seems to be more related to the packing fraction of the granular material. The higher the packing fraction, the higher the angle. This interpretation is in agreement with pile stability reported in very compact granular materials.

\section{References}

[1] E. Espiritu, A. Kumar, A. Nommeots-Nomm, J. Lerma, M. Brochu, Powder Technol. 366, 925 (2020)

[2] H. Henein, J. Brimacombe, A. Watkinson, Metall. Mater. Trans. B 14, 191 (1983) 
[3] J. Mellmann, Powder Technol. 118, 251 (2001)

[4] R. Yang, A. Yu, L. McElroy, J. Bao, Powder Technol. 188, 170 (2008)

[5] R. Brewster, G. Grest, A. Levine, Phys. Rev. E 79, 011305 (2009)

[6] G. Lumay, F. Boschini, K. Traina, S. Bontempi, J.C. Remy, R. Cloots, N. Vandewalle, Powder Technol. 224, 19 (2012)

[7] D. Höhner, S. Wirtz, V. Scherer, Powder Technol. 235, 614 (2013)
[8] D. Höhner, S. Wirtz, V. Scherer, Powder Technol. 253, 256 (2014)

[9] D. Höhner, S. Wirtz, V. Scherer, Powder Technol. 278, 286 (2015)

[10] G. Lu, J. Third, C. Müller, Chem. Eng. Sci. 127, 425 (2015)

[11] J. Olson, M. Priester, J. Luo, S. Chopra, R. Zieve, Phys. Rev. E 72, 031302 (2005)

[12] R.A. Bagnold, Proc. R. Soc. A. 295, 219 (1966)

[13] Q. Chen, H. Yang, R. Li, W. Xiu, R. Han, Q. Sun, V. Zivkovic, Powder Technol. 364, 1039 (2020) 